Hope College

Hope College Digital Commons

Faculty Publications

7-1-2019

\title{
Tuned Photomechanical Switching of Laterally Constrained
}

\section{Arches}

\author{
Matthew L. Smith \\ Hope College, msmith@hope.edu \\ J Gao \\ University of Pittsburgh \\ A A. Skandani \\ University of Pittsburgh \\ N Deering \\ Hope College \\ D H. Wang \\ Wright-Patterson Air Force Base
}

See next page for additional authors

Follow this and additional works at: https://digitalcommons.hope.edu/faculty_publications

Part of the Structural Materials Commons

\section{Recommended Citation}

Repository citation: Smith, Matthew L.; Gao, J; Skandani, A A.; Deering, N; Wang, D H.; Sicard, A A.; Plaver, M; Tan, L-S; White, T J.; and Shankar, M R., "Tuned Photomechanical Switching of Laterally Constrained Arches" (2019). Faculty Publications. Paper 1483.

https://digitalcommons.hope.edu/faculty_publications/1483

Published in: Smart Materials and Structures, Volume 28, Issue 7, July 1, 2019, pages 075009-. Copyright (C) 2019 IOP Publishing.

This Article is brought to you for free and open access by Hope College Digital Commons. It has been accepted for inclusion in Faculty Publications by an authorized administrator of Hope College Digital Commons. For more information, please contact digitalcommons@hope.edu. 


\section{Authors}

Matthew L. Smith, J Gao, A A. Skandani, N Deering, D H. Wang, A A. Sicard, M Plaver, L-S Tan, T J. White, and M R. Shankar 


\title{
Tuned photomechanical switching of laterally constrained arches
}

\author{
M. L. Smith ${ }^{1 *}$, J. Gao ${ }^{2}$, A. A. Skandani' ${ }^{2}$, N. Deering ${ }^{1}$, D. H. Wang ${ }^{3}$, A. A. Sicard ${ }^{1}$, M. \\ Plaver $^{1}$, L.-S. Tan ${ }^{3}$, T. J. White ${ }^{3}$ and M. R. Shankar ${ }^{2}$ \\ ${ }^{1}$ Department of Engineering, Hope College, Holland, Michigan, USA \\ ${ }^{2}$ Department of Industrial Engineering, University of Pittsburgh, Pittsburgh, Pennsylvania, \\ USA \\ ${ }^{3}$ Materials and Manufacturing Directorate, Wright-Patterson Air Force Base, Dayton, $\mathrm{OH}$, \\ USA
}

E-mail: msmith@hope.edu

\begin{abstract}
Laterally constrained arches driven between stable states by light, represent a unique space for compliant mechanism design. Exploiting mechanical multistability can overcome limitations of functional photomechanical actuators, which include limited repeatability, actuation speed, and positioning characteristics. Here, the addition of lateral constraints to an elastic bistable arch system is proposed as a method for toggling between bifurcated states by controlling the location of actinic irradiation. This approach expands the design space for photomechanical, mutistable structures and actuators. Arch behavior as a function of system parameters is simulated, including conditions leading to multistability. An experimental demonstration and exploration of a constrained photomechanical arch is also presented. It is expected that the concepts presented here could lead to innovations in areas such as energy harvesting, soft robotics, and multistable architected materials.
\end{abstract}

\section{Introduction}

$\mathrm{Bi}$ - and multistable elements hold potential for a variety of applications in areas such as motion stabilization, mechanical or thermal energy harvesting, actuators, sensors, morphing surfaces and structures, and robotics [1-4]. These elements are a subset of a broader class of device elements termed compliant mechanisms. Compliant mechanisms make use of flexible components, rather than discrete, rigid links, and are designed to perform specified functions which combine both motion and force control [5]. These mechanisms are commonly employed in nature for the purpose of enhanced kinematics or accelerated motion (e.g. cicada ribs, hummingbird beaks, or the Venus flytrap [6-8]). By replacing multiple rigid elements with a smaller number of flexible components, compliant mechanisms offer integral, easy-to-fabricate structures with advantages that include, reduced wear and maintenance, lower overall device weight, and novel design strategies (e.g. in energy harvesting and energy dissipation applications) [1,5,9].

Realizations of these mechanisms using photomechanical materials, which convert light into mechanical work, is particularly intriguing because of the opportunity to induce spatially resolved, wireless actuation that can be actively controlled or designed to respond to environmental conditions. While the development of photochemically driven light responsive materials has been the focus of intense investigation [10-14], the utilization of these materials in functional compliant mechanisms [5] for smart structures and devices has not been extensively explored (see Ref. [15-18] for some notable exceptions). 
In particular, most photomechanical realizations of these materials have involved standard cantilever bending configurations as proof-of-concept, light to work demonstrations. In cantilever configurations, these materials often exhibit slow actuation rates ranging from 10's of seconds to minutes, unless large laser intensities on the order of hundreds of $\mathrm{mW} / \mathrm{cm}^{2}$ are used [19]. Photomechanical cantilevers also typically have low flexural stiffness and display poor reproducibility in tip positioning from sample to sample.

These challenges can be partially addressed through mechanical design. For example, a simple arch configuration (Figure 1a, both ends clamped) offers distinct advantages over cantilevers, such as, precise, discrete positioning and the ability to hold an equilibrium position without further energy input. An additional significant feature of this configuration is the potential for rapid motion (snap-through or snapbuckling) between equilibrium states, due to elastic instability. Bistability and snap-buckling have attracted the interest of a wide range researchers, and these phenomena have been utilized in many developing applications, including a ventricular assist device [20], jumping robotic locomotion [21], and passive flow control in microfluidics [22].

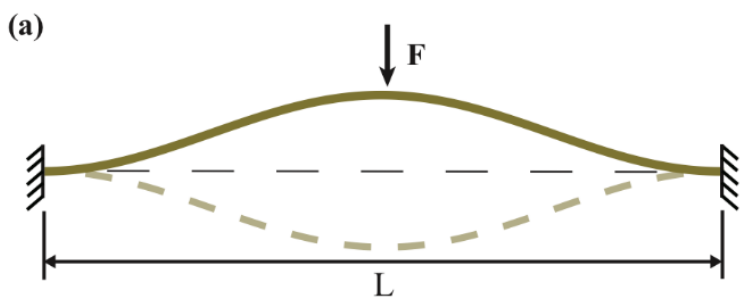

(b)

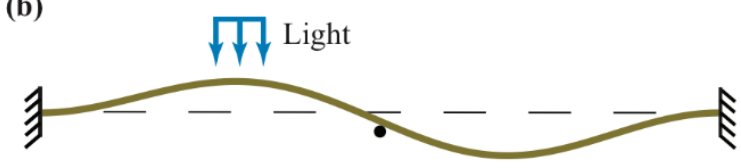

(c)

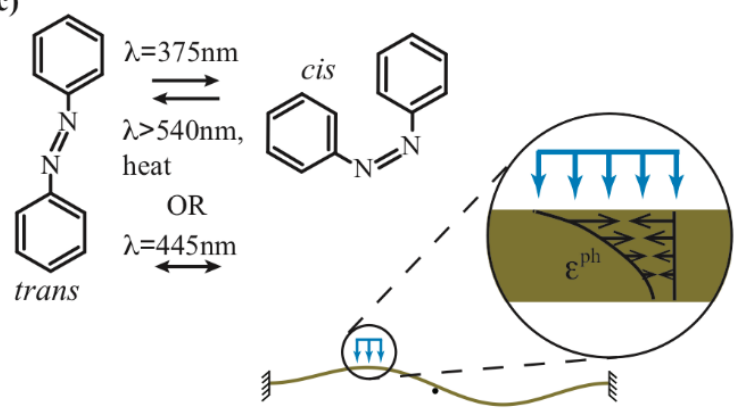

Figure 1. (a) Schematic of the material strip buckled into a simple arch with width $\mathrm{L}$ and no rigid constraint. Classical snap-buckling induced by a lateral force is indicated. (b) Buckled, photo responsive material strip with rigid constraint. Lateral loading is produced by light. (c) Azobenzene isomers and a schematic of a photoinduced strain gradient in the material strip.

Generally speaking, the qualitative behavior of a simple arch, clamped at both ends (clamped-clamped), remains relatively unchanged while varying material stiffness, beam thickness, and arch width. However, some recent studies have revealed more complex arch behavior by modulating boundary conditions, exploiting symmetry breaking (via arch material construction; bifurcations driven by boundary conditions), or applying external constraints. For example, Plaut and Virgin [23] investigated snap-through of a buckled, flexible strip subjected to rotations at the boundaries. Gomez et al. [24] used an arch with clamped-clamped boundary conditions, where one end was also rotated at an angle, to investigate a critical slowing down of snap speeds near the point of instability. Sano and Wada [25] studied a buckled beam with one clamped end and the other hinged. They broke the symmetry in the system by moving the clamped end laterally and discovered interesting hysteretic but reproducible behaviors, including snap buckling into monostable configurations. Furthermore, Arena et al. [26] exploited symmetry breaking in a composite layup to realize a passively adaptive air inlet featuring "super elastic" monostability in which the structure snaps into a closed position at high air velocities and automatically snaps back open when the air velocity is lowered to a critical level.

Contact with external constraints to exploit non-linear responses of slender geometries has also attracted significant interest. Recent studies on mechanical energy harvesting devices have highlighted the use of external point loads and discrete rigid constraints as a means to tailor and broaden the system response of buckled and arch-like structures $[27,28]$. Sano et al. studied the interaction of the free ends of 
singly clamped elastic strips with rigid substrates [29]. In addition, contact of thin structures with rigid constraints have application to Coiled Tubing technology in the petroleum industry [30], rope coiling [31], and pattern formation in thin shells with inner mandrel constraints [32]. The buckling and collapse behavior of thin beams experiencing contact with a rigid, frictional, lateral constraint has also received some past research interest $[33,34]$.

In this work, we explore the use of discrete, rigid constraints (Figure 1b) as a means to produce qualitative changes in arch behavior. In addition, these constraints provide additional parameters that can be used to tune the mechanical response. In the following sections, we first present numerical simulations that outline the basic operation of an arch with a single rigid constraint followed by model predictions for tuning this type of actuator. We discuss opportunities for generating wireless, multi-stable compliant structures by introducing more than one constraint. Finally, the operation of a photomechanical actuator with a single constraint is demonstrated experimentally.

\section{Methods}

\subsection{Numerical Modeling, Stationary Simulations}

The photomechanical materials considered herein are glassy polyimides functionalized with azobenzene derivatives [35]. Azobenzenes are chromophores that undergo a molecular contractions during isomerization from a rod-like trans state to a bent cis state when irradiated with certain wavelengths of light (Figure 1c) [36]. Irradiation induces strain in the material. As light interacts with the material it is absorbed by the chromophores. Because the chromophores are evenly distributed throughout the material, the light's intensity attenuates as it penetrates into the thickness. Lower light intensity in the material bulk away from the irradiated surface leads to lower isomerization rates, and ultimately, a gradient in strain through the material thickness. It is this gradient that produces bending in cantilever and arch structures fabricated from such photoactive materials.

A crucial feature of the azobenzene molecule is that its absorption of light is axis-selective, that is, it absorbs light most strongly along its transition dipole (in the trans state this is roughly along the molecular axis). In a material with isotropic alignment, polarized light can be used to determine the direction of material contraction since predominantly the azobenzenes parallel to the light polarization will isomerize. Either uv or blue light can be used to trigger a photomechanical response, however the exact molecular level mechanisms leading to deformation are different depending on the light source [12]. In this modeling effort, we focus primarily on the macroscopic structural response given an assumed exponential strain gradient in response to the light. In this manner, the effect of irradiation on the material can be thought of as resulting in a local change in natural length, similar to thermal expansion or contraction.

A reasonable approach for modeling the thin photomechanical material strips considered in this work, is to use a one-dimensional gradient beam model. This approach has been used, for example, to study thermal loadings in functionally graded material beams [37,38] and for bending [39-41] and snap-through $[42,43]$ in photomechanical systems. The contact conditions along with the photo induced loading can make

the analysis challenging and such a one-dimensional system is not pre-coded in commercial finite element software. An alternative approach that is relatively convenient is to treat the material strip as a planar, linear 
elastic solid. We employ this technique here, performing stationary simulations in COMSOL Multiphysics. Coordinates of material points in the reference configuration (Figure 2a) are given by $X \in$ $\left[0, L_{o}\right]$ and $Y \in[-h / 2, h / 2]$, where $L_{o}$ and $h$ are the original length and thickness of the linear elastic solid, respectively. Displacements of material points are given by the two dimensional vector, $\boldsymbol{u}(X, Y)$. The arch is subjected to clamped boundary conditions at both ends, such that, displacements and rotations are restricted.

The elastic strain is given by $\varepsilon^{e l}=\varepsilon-\varepsilon^{s}$, where $\varepsilon$ is the total strain and $\varepsilon^{S}$ is any contribution to the strain due to non-mechanical stimulus, such as, the photomechanical effect. The constitutive model is given by $\boldsymbol{S}=\boldsymbol{C}: \varepsilon^{e l}$, where $\boldsymbol{S}$ is the second PiolaKirchhoff stress tensor, $\boldsymbol{C}$ is the stiffness tensor, and $\varepsilon^{e l}$ is the Green-Lagrange strain tensor. Material parameters that are entered into the stiffness tensor using the COMSOL interface are Young's Modulus, $E$ and Poisson's ratio, $\nu$. Since the light is considered to be polarized parallel to the $X$ direction, the only photoinduced strains are assumed to be of the form $\varepsilon_{X}^{S}=$ $\varepsilon_{X}^{p h}=I_{o}(X) \beta e^{-(h / 2-Y) / d}$ and $\varepsilon_{\perp}^{s}=\varepsilon_{\perp}^{p h}=-v^{p h} \varepsilon_{X}^{p h}$, where $\varepsilon_{\perp}^{p h}$ is the induced strain in the direction perpendicular to the $X$ direction due to a photo Poisson-like effect with proportionality constant $v^{p h}[44,45]$. Further, $\beta$ is the proportionality constant between photostrain and light intensity and is termed the photocompliance, $d$ is the attenuation length of the light for the azoPI films, and $I_{o}(X)$ is the light intensity at the incident surface, which can vary in the $X$ direction. Here we assume the laser is collimated and the intensity profile in the $X$ direction is sufficiently approximated by a boxcar function (cf. Figure $2 \mathrm{a}$ ). The light induced, inelastic strain field is implemented in COMSOL using the initial strain subnode.

In a representative simulation, first, parameters such as the pin placement and laser focus point are set. Friction free contact surfaces are assigned between the bottom of the strip and the fixed pin. In the first simulation step, the material strip is buckled by displacing the right boundary condition inward so that the width of the arch is length $L$. A temporary, downward body force is applied on the right or left side of the strip so that it buckles into the rigid pin and forms a constrained arch (cf. Figure 1b). Once the strip is in the buckled configuration, the body force is removed, and a parameter sweep is performed in which the vertical displacement of a material point located at $X_{f}$ (the laser focus point) is varied and equated to the actual displacement through a global equation node. Though the actual light intensity is known in experiment, the transduction of that intensity into a photostrain gradient is not instantaneous and so the actual photostrain with time is not known. In a dynamic simulation the population of photo active molecules could be modeled to gain additional understanding of when the gradient reaches a point critical for snapthrough [46,47]. For the stationary solutions here, the incident light intensity is treated as an extra degree of freedom and the software solves the system of stationary equations of motion along with the global equation to yield the simulation results. In essence, a photo stationary state is assumed at each incident intensity computed. The critical incident intensity can be identified by locating turning or limit points on the computed equilibrium path. 


\subsection{Dynamic modeling}

All dynamic simulation models were created using the commercial finite element software, LS-DYNA. LSDYNA was selected for its ability to effectively simulate highly nonlinear dynamic problems involving contact conditions. In particular, it features contact detection involving plates and shells which is an advantage for computational efficiency. A tradeoff in this approach is that the through thickness strain gradient is not readily implemented in LS-DYNA. Therefore, out of expedience the arch was modeled as an elastic bilayer plate, while the pins were modeled as rigid solid cylinders. A region of the top layer was given a material definition that included a negative coefficient of thermal expansion in order to simulate the light irradiation. The bottom layer contained no thermally responsive region. All other material properties for the two layers were identical and matched those used for the stationary simulations (Table S1).

We follow Ref. [48] in implementing the bilayer approach. A relationship relating the active bilayer thickness $H$, stimuli induced strain in the active bilayer $\varepsilon^{b i l}$, and the photostrain at the incident surface in the gradient model $\varepsilon_{o}^{p h}$, can be developed by equating the internal resultant forces in the $X$ direction produced by the gradient model and the bilayer model, and by equating the internal resultant moments about the $Z$-axis for the two models. This relationship as derived in reference [48] is restated in equations (1) and (2) for the reader's convenience:

$H=\frac{2\left[d\left(e^{h / d}-1\right)-h\right]}{e^{h / d}-1}$,

$\varepsilon^{b i l}=\frac{d \varepsilon_{o}^{p h}\left(e^{h / d}-1\right)^{2}}{2 e^{h / d}\left[d\left(e^{h / d}-1\right)-h\right]}$,

Additional details related to the derivation of equations (1) and (2) are given in the supplemental information, Section S2.

A surface to surface contact type was used between the pins and plate. The arch was buckled into the pin first, in all simulations. Once buckled, both ends of the plate had rotation and translation restricted in all directions. After the beam attained a stable state, the temperature was ramped to mimic the effect of irradiation with light, and the thermally responsive section began to deform. Deformation was relatively slow at first until a point of instability was approached and then rapid snap-through commenced. The thermally responsive region can be relocated in order to transition the arch to different stable positions. After the new stable states were attained the temperature was reduced to the reference state and the arch relaxed so that no non-mechanical strain remained.

\subsection{Experimental Details}

Cross-linked azobenzene functionalized polyimide (AzoPI) films with the chemical structure shown in Figure $2 b$ were synthesized following previously reported procedures $[17,35]$. Films contained $30 \mathrm{~mol} \%$ azobenzene units and the specific material was designated as X-ppp-6FDI in prior publications [35,46]. Thin strips, having nominal dimensions of $b=1 \mathrm{~mm}$ wide and $h=15 \mu \mathrm{m}$ thick, were cut from solution cast films. The strips were clamped so that the average length in the unbuckled configuration $\left(L_{o}\right)$ was approximately $56 \mathrm{~mm}$ (Fig. 2a) unless otherwise noted. The displacement of one end of the strip was 
controlled by a linear stage with a micrometer while the other end was fixed. The pin used to constrain the arch motion was fixed to a vertical stage with micrometer and had a diameter of $0.7 \mathrm{~mm}$ (Fig. $1 \mathrm{~b}$ and $2 \mathrm{a}$ ). In a typical experiment, one end of the strip was displaced inward slightly in order to induce buckling. If necessary, the buckled arch was manually biased downward so that it contacted the pin. The sample was then irradiated with a $375 \mathrm{~nm}$ wavelength laser. The diameter of the laser spot on all samples was $3 \mathrm{~mm}$ with an intensity of $100 \mathrm{~mW} / \mathrm{cm}^{2}$ based on measurements from a Thorlabs S302C thermal power sensor. An Aven Mighty Scope camera was used to record the light induced deformations at 7 frames/s. If the sample was to be tested again (e.g. in order to examine back and forth snap-through) it was irradiated with visible light from a $150 \mathrm{~W}$ fiber optic illuminator with a halogen bulb (Cole Parmer) in order to drive cis isomers back to trans isomers. Images were processed using the open source software ImageJ [49] and a custom code written in MATLAB.

\section{Results and Discussion}

\subsection{Numerical predictions}

In the following sections, results from both the stationary and dynamic simulations are presented. An overview of representative arch behavior is given in section 3.1.1. System output, such as, rotational travel at the midpoint and stroke length as certain parameters are varied is shown in section 3.1.2. Finally, multistable configurations with 1 to 4 rigid constraints are discussed briefly in section 3.1.3. The stationary simulations were used to generate the results in sections 3.1.1 and 3.1.2, while the dynamic simulations were used to produce the configurations shown in section 3.1.3, in order to demonstrate the arch could be dynamically transitioned between states using only non-mechanical stimulus.

\subsubsection{Constrained Arch Operation}

The basic operation of the photomechanical, constrained arch is demonstrated in Figure 3 using the stationary model in COMSOL. First, the arch is buckled so that it comes into contact with the rigid pin. A light induced strain gradient is applied to the arch and a path of equilibrium solutions is traced. Key equilibrium configurations (Fig. 3a,b) are numbered (1)-(6). Configuration (1) of the strip approximates the second Euler buckling mode shape, but with the pin offset downward (the top of the pin is non-collinear with the clamps) the strip is in stable equilibrium (Figure 3a,c). The pins were offset in a similar manner in all subsequent simulations. For this particular simulation, the right hand side of the arch was biased downward during buckling in order to begin the cycle with configuration (1). Next, the left hand side of the structure is irradiated (i.e., the expected contractile photostrain gradient is simulated) with increasing light intensity, tracing out a path of stable equilibria from (1) to (2). When the left side of the arch, which is concave down, is irradiated from above with growing intensity, the effect is to produce an increasingly concave up intrinsic curvature (via the photo induced strain gradient) such that the arch begins to flatten, then turn upward, and ultimately reach a point of instability.

Indeed, a turning or limit point occurs at (2) indicating a transition from stable to unstable equilibria. In the physical system a rapid, dynamic jump is expected to occur from (2) to another stable equilibrium point at (3) (see Fig. S1 for a supporting dynamic simulation result). The material is assumed to be completely elastic (i.e. no photofixing or strain persistence is considered) so, as the light intensity is decreased from (3) to (4) the material strip returns to a configuration near the second Euler buckling mode shape (only now with the left hand side buckled downward).

To return to the original buckled configuration at (1), the right hand side of (4) is irradiated. Again a path of stable equilibria (Fig. 3b) is traced out from (4) to (5). The same material point that was used in Fig. 
3a is used for the normalized displacement in Fig. 3b, however the irradiated region is switched to the opposite side of the structure. Another limit point is encountered at (5) and a dynamic release of elastic energy is expected to occur as the system rapidly transitions from (5) to (6). Finally, as light intensity is reduced the system is restored to the original buckled state at (1). The ability to toggle the actuator between the laterally bifurcated states using irradiation from the same side is a characteristic of value to the design of light-actuated mechanisms that harness the impulse from the snap-through for functional manipulation.

(a)

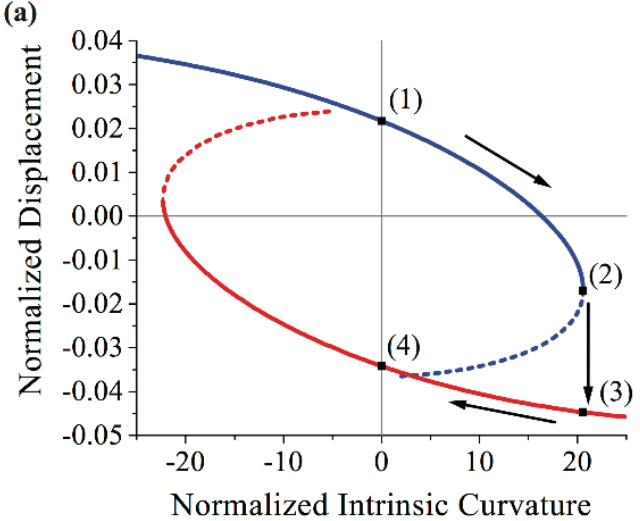

(c)

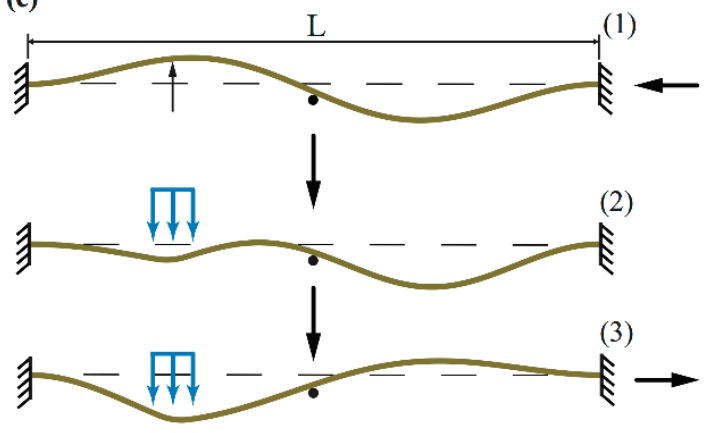

(b)

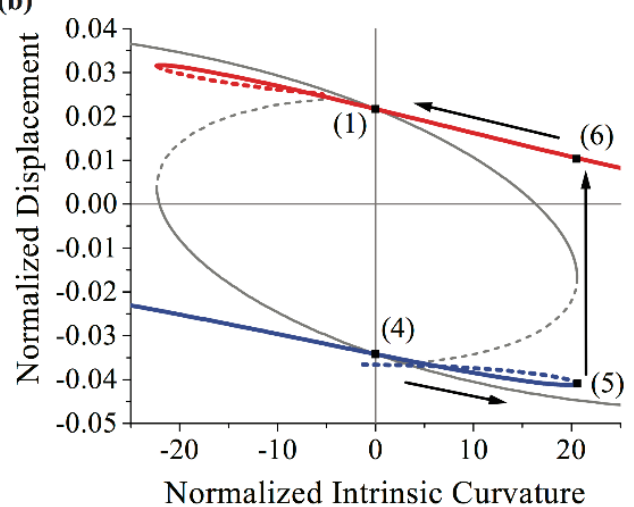

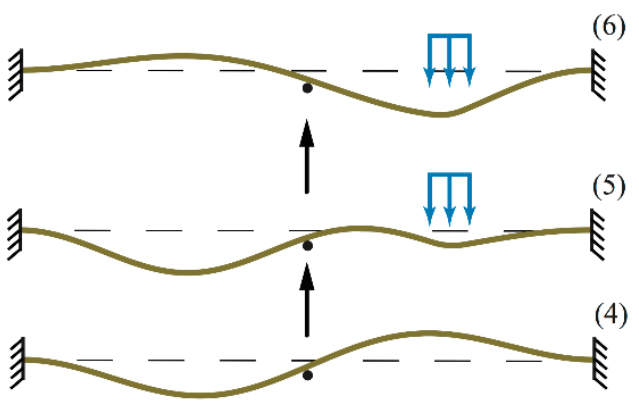

(6)

Figure 3. (a) Equilibrium solution paths for the constrained arch showing the first half of the irradiation cycle. Solid and dashed lines signify stable and unstable equilibria, respectively. (b) Equilibrium solution paths for the constrained arch during the second half of the irradiation cycle, which returns the arch to its original configuration. The thin gray lines show the equilibrium solutions obtained in the first plot. (c) Evolution of the constrained arch configurations for steps (1)-(6). Actual simulated shapes are shown. Note, the small black upward arrow in part (c), configuration (1) indicates the material point whose normalized vertical displacement $\left(u_{2} / L_{o}\right)$ is plotted in both $(a)$ and $(b)$. A normalized intrinsic curvature measure $\left(b d^{2} L_{o} \beta I_{o} / I_{z z}\right)$ is plotted on the horizontal axis where $b$ is the width of the material strip and $I_{z z}=b h^{3} / 12$ is the area moment of inertia of the strip cross section about the neutral axis [43].

\subsubsection{Tuning Arch Parameters}

One appealing aspect of a constrained arch is the ability to tune system behavior using parameters such as the laser focus point, pin position, or end displacement (Fig. 4a). This tuning allows for versatile system design of stroke length, center point rotation, critical light intensity required for actuation, and the emergence and control of multistability. Examples of simulated system behavior for various parameter values are given in Figure 4b-e. Figure $4 \mathrm{~b}$ shows how end displacement effects stroke length (maximum lateral displacement of the arch) and rotational travel of a vector normal to the strip at its center point. The 
structure can readily be tuned to have maximum linear stroke lengths of approximately $2-12 \%$ of the original length of the material strip and rotations of 5-60 degree. Rotational travel is a new feature that appears with the implementation of lateral constraints and is of interest in applications such as light beam steering and for translation of linear motion to rotational motion through ratcheting mechanisms. Of course, as the end displacement increases so does the critical photostrain required for snap-through, similar to a single arch design (Fig. S2) [42].

Figure $4 \mathrm{c}$ demonstrates how horizontal positioning of the pin can be used to generate asymmetric device operation, in this case, asymmetric stroke length. The critical photostrain required to switch states also becomes asymmetric. Irradiating the short side (left side, Fig. $4 \mathrm{c}$ inset) to initiate snap-through requires more photo induced strain than irradiating the long side. A feature of this design motif is that a single actuator can operate at two different stroke lengths and have the potential to hold static position under two different maximum force levels. The critical strain and rotational travel can also be tuned by varying the vertical pin displacement (Fig. 4d). The lowest pin positions also change the qualitative nature of the snap from primarily vertical motion to more of a horizontal shifting of the arch's minimum position (characterized by low rotational travel), a motion that might be useful for mass transport (cf. Fig. 4a, lower left schematic).

Characteristics of the irradiating light, including focus point position and light beam width have a significant influence on constrained arch operation (Fig. 4e). The distance between the left end of the arch and the pin is given by $p_{x}$. The critical photostrain required for snap through (left-side up to left-side down) was plotted as a function of the fraction of the region between the pin and clamp covered by the laser beam $\left(X_{w} / p_{x}\right)$, where $X_{w}$ is the width of the laser beam. The critical photostrain is minimized for light beam coverage around $55 \%$ of the distance between the clamp and the pin, where strains of about $1 \%$ on the irradiated surface are required for snap-through. Critical strain remains relatively low $(<2 \%)$ until the light beam width shrinks below $X_{w} / p_{x} \approx 0.15$.

Perhaps of greater interest is the changing character of the snap-through as the focus point of the light is changed (Fig. 4e, triangles). In this case, the light width was fixed at $X_{w} / p_{x}=0.108$ which is the value used in the experimental demonstrations. The critical photostrain has a minimum near $X_{f} / p_{x}=0.54$ (nearly centered between the pin and clamp) and a maximum near $X_{f} / p_{x}=0.76$. However, below $X_{f} / p_{x}=0.44$ a third stable state manifests itself. Arches irradiated in this range lose contact with the pin and take the conformation of a single arch buckled upward. This finding is particularly interesting because all three stable states (left-side down, left-side up, and total strip up) can be attained reversibly by irradiating the arch from above by simply changing the focus point of the light. The predicted multistability of these arches are now explored briefly in the next section. 
(a)

Tune support geometry

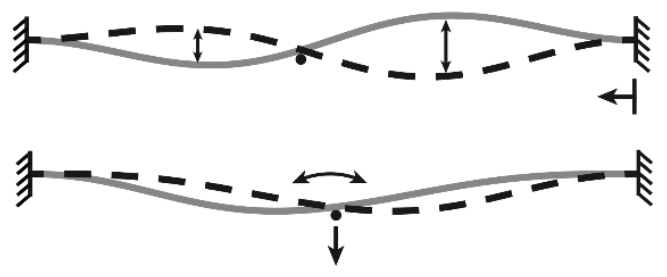

(b)

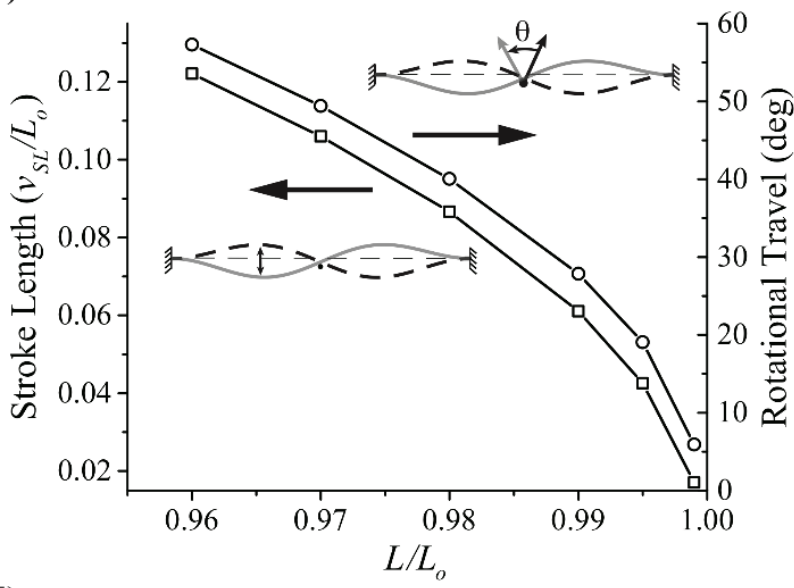

(d)

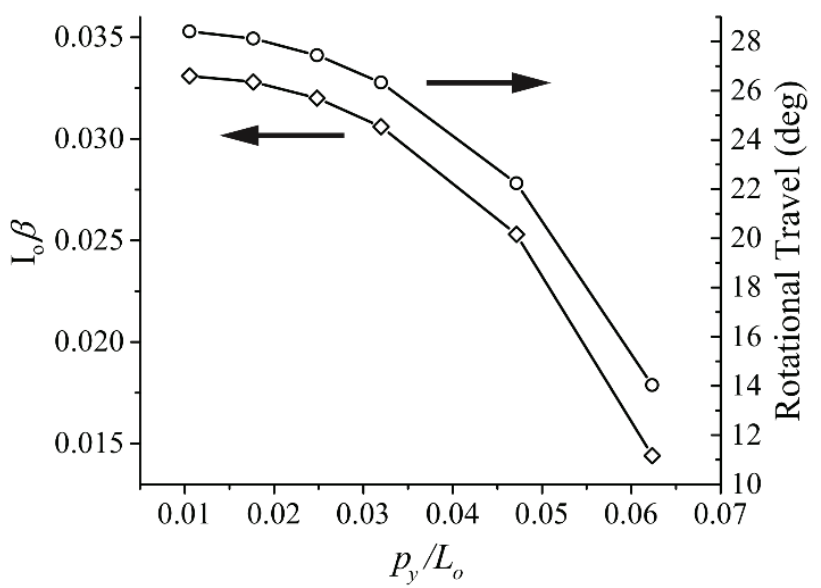

Tune light characteristics

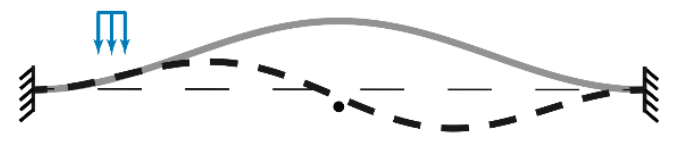

(c)

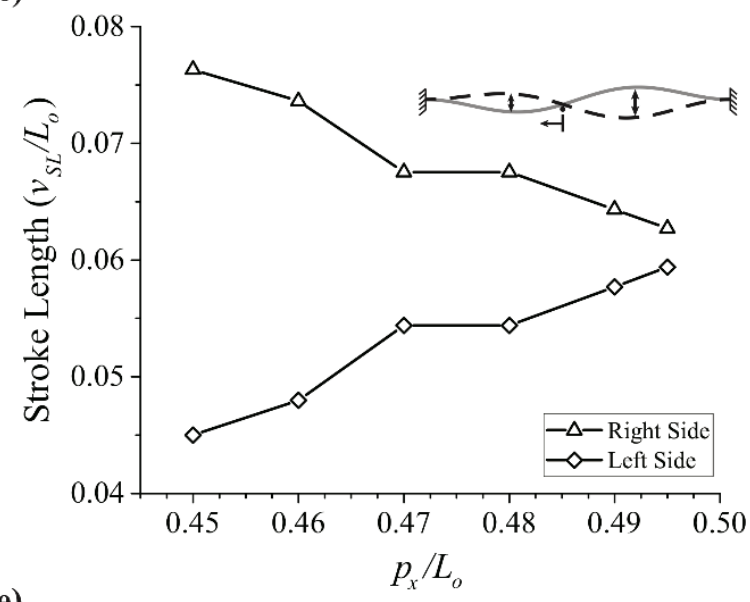

(e)

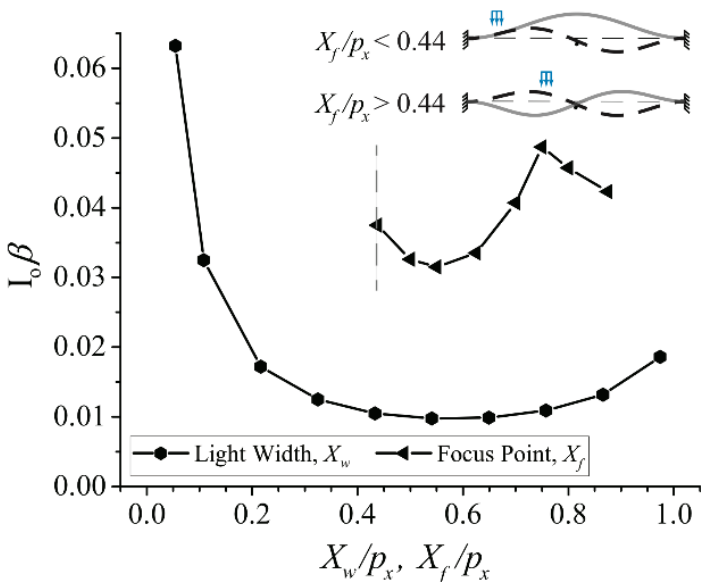

Figure 4. (a) Constrained arch behavior can be tuned through a variety of parameters including pin position, end displacement, and focus point or width of the irradiating light beam. (b) End displacement affects the stroke length (squares) of the arch and the rotational travel of the normal vector at the center of the material strip (circles). Here the pin is perfectly centered between the two end points of the strip. The maximum lateral displacement of the arch from the initial buckled state to the snapped through state is represented by $v_{S L}$. (c) Maximum stroke length of the arch on the left and right side of the pin as a function of horizontal pin position. As the pin position is shifted to the left, the right-side stroke length increases (triangles), while the left-side stroke length decreases (diamonds). (d) Critical photostrain (diamonds) and rotational travel (circles) is plotted as a function of the normalized vertical pin position (cf. Fig. 2a). The pin was fixed at the horizontal midpoint of the arch. (e) Critical photostrain is plotted as a function of the normalized width of the light beam $\left(X_{w} / p_{x}\right.$, circles; $X_{f} / p_{x}$ fixed at 0.505$)$ and as a function of the normalized focus point of the light beam $\left(X_{f} / p_{x}\right.$, triangles; $X_{w} / p_{x}$ fixed at 0.108).The pin is once again centered. End displacement $L / L_{o}=0.99$ was used for each simulation in $(c),(d)$, and $(e)$. All results were generated with a stationary model in COMSOL. 


\subsubsection{Multistability through Constraints}

Rigid, lateral point constraints enable the design of photomechanical actuators that can be reversibly switched to multiple stable states. In the cases considered here (Fig. 5a-d) the number of states attained is always $N+2$, where $N$ is the number of rigid constraints. Further, each of the states can be accessed, in principle, by irradiating the arch from above by judicious placement of the light beam focus point. For example, in the two pin case (Fig. $5 b)$ the two downward positions (solid black and dashed black lines) can be toggled by alternately shining light in positions (1) and (2). To reach the upward-left (black dash-dot line) configuration the solid black arch must be irradiated at position (3). Irradiation at (3) produces a concave up curvature that makes a buckled up configuration more favorable and also induces an asymmetry that pulls the arch to the upward-left position. Likewise, to reach the upward-right configuration (gray dashdot line) the black dashed arch must be irradiated at position (4). To restore contact with the lower pin, the black dash-dot configuration can be irradiated
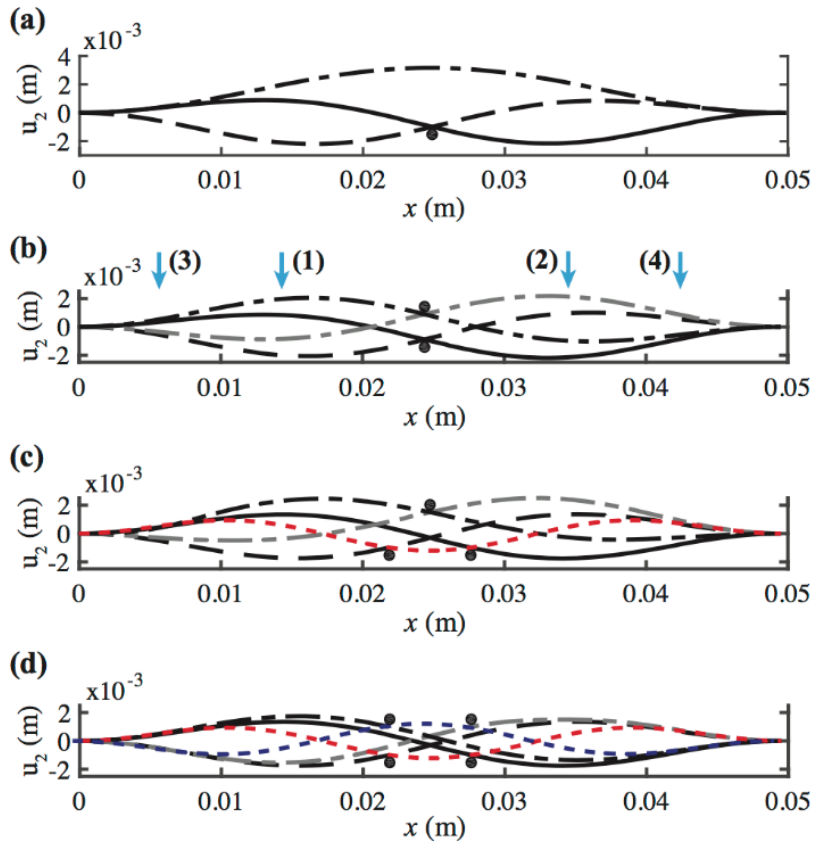

Figure 5. Demonstration of attainable stable states for a variety of rigid pin placements: (a) 1 pin, 3 states (b) 2 pins, 4 states (c) 3 pins, 5 states (d) 4 pins, 6 states. Note, all contacting surfaces of the pins are non-collinear with the clamps. All states were simulated in LS-DYNA. at position (1) or the gray dash-dot configuration can be irradiated at (2). These kinds of unimorphic, multistable compliant mechanisms should be simple to construct and control. The realization of multiple stable positions holds promise for complex actuation applications, mechanical logic/memory, and to harvest and harness energy from ambient sources. In addition, it is also possible to combine discrete and continuous constraints (Fig. S3) to expand functionality to areas such as mass transport. Exploration of multistable mechanisms made from different responsive materials with a variety of discrete, rigid lateral constraint configurations, should be the subject of future investigation.

\subsection{Photomechanical demonstration with a single constraint}

To demonstrate the left-right coupled snap behavior of photomechanical, constrained arches, rectangular azoPI strips $\left(L_{o}=56 \mathrm{~mm}, b=1 \mathrm{~mm}, h=15 \mu \mathrm{m}\right)$ were cut from a bulk sheet. Each strip was clamped at both ends and compressed until the desired arch width to original length ratio $\left(L / L_{o}\right)$ was obtained. In the representative case shown in Figure 6a, the arch starts with the left side up and the right side down (lower right corner). The sequence of irradiation and snap-through is analogous to the simulated results in Figure 3. Note that the pin is located with the top surface collinear with the clamps. As a result of this location, the strip starts out very near the second Euler buckling mode, which should be unstable. However, in all experiments the buckled strip remains in contact with the pin and never evolves into a first mode shape, buckled upward. During handling the thin strips exhibit what appears to be electrostatic attraction to surrounding surfaces and likely the same driving force secures the strip to the pin. 
(a)

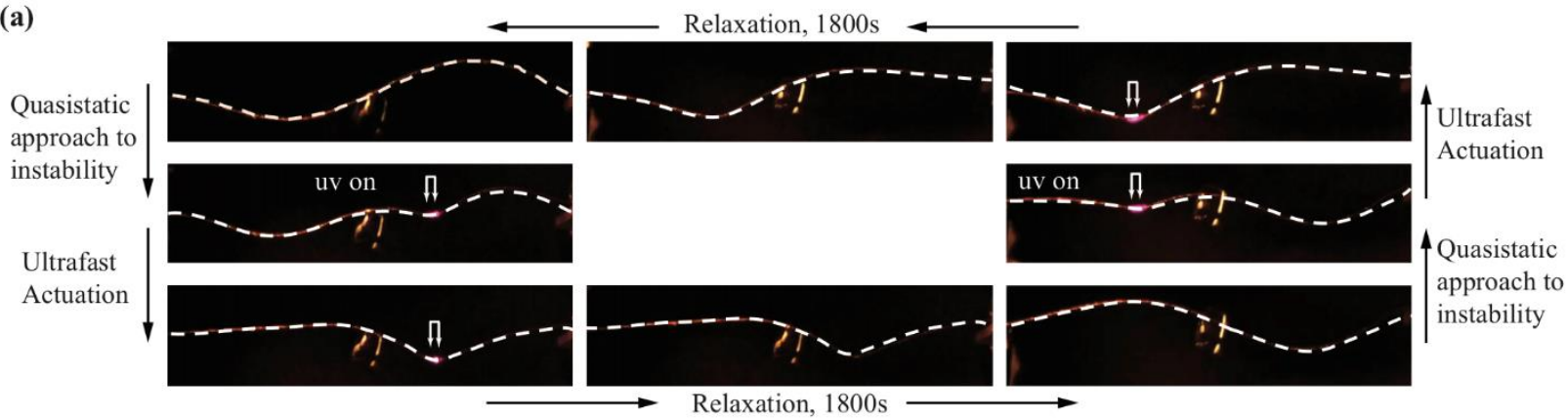

(b)

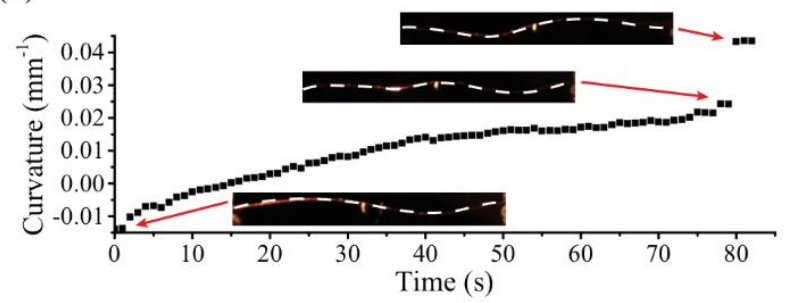

(c)

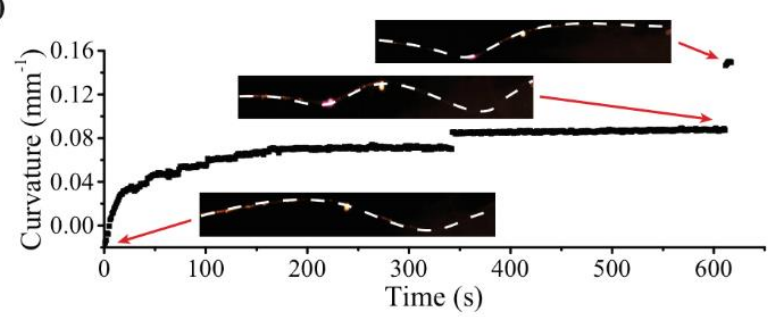

Figure 6. (a) Experimental demonstration of a complete actuation cycle using unidirectional irradiation $\left(L / L_{o}=\right.$ 0.975, pin located $200 \mu \mathrm{m}$ below reference state). Dashed lines were overlaid on the arch to aid visualization. (b) Curvature vs. time plot for $L / L_{o}=0.99$ with the top of the pin collinear with the two clamped ends. The quasistatic approach to instability is shown followed by snap-through at 80 seconds. (c) Curvature vs. time plot for $L / L_{o}=0.96$ with the top of the pin collinear with the two clamped ends.

Working around Figure 6a in a counter clockwise fashion (starting in the lower right corner), the center of the left side of the strip is irradiated with $375 \mathrm{~nm}$ light, which leads to an increase in stored elastic energy. At the point of elastic instability, the arch undergoes ultrafast actuation or snap-through ( 10ms duration), such that the left-side transitions to the "down" state and the right side to the "up" state, simultaneously. During the relaxation stage the sample is irradiated with visible light $(>500 \mathrm{~nm})$ in order to drive residual cis isomers back to the trans state and relieve the photo induced strain. A feature of this material system that was not captured in the simulations (for simplicity), is the kinetics of both the azobenzene photo reaction and the relaxation dynamics of the material after snap-through. The right side of the arch is then irradiated with the $375 \mathrm{~nm}$ source, leading again to snap-buckling. Relaxation with visible light returns the arch to its original configuration. After a few cycles $(\sim 5)$ the snap-buckling effect on at least one side often ceases. However, the arch still transitions to both states with half of the cycle resulting in snap-through and the other half of the cycle exhibiting a slower transition. The complex history of photokinetics and polymer relaxation dynamics during cycling likely leads to residual strains that are not completely resolved by irradiating with visible light. To completely reset the material, it may need to be thermally processed.

The change in curvature during irradiation is shown for two representative cases in Figure $6 \mathrm{~b}$ and $\mathrm{c}$. Some samples experience one or more intermediate small jumps in curvature before a final snap through (as seen at approximately 340 seconds in Figure 6c). These intermediate jumps could be a frictional stickslip effect from the pin, from defects in the strips, or possibly jumps into quasi-stable mode shapes.

The behavior of constrained and non-constrained arches is compared in Figure 7. The latency time $\left(T_{c r}\right)$ or time to trigger snap-through was recorded for different light positions and end-displacement ratios. In cases where there was more than one jump, the time of the final jump was assigned as $T_{c r}$. In both the constrained and non-constrained cases, $T_{c r}$ increases as the arch is buckled more severely $\left(L / L_{o}\right.$ decreases). 
As expected, the unconstrained arch exhibited symmetric behavior for light focused on the left and right sides of the arch midpoint (triangles and circles in Fig. 7a) with the greatest latency times occurring for light focused directly in the middle of the arch (squares). Stationary simulations of a nonconstrained arch using COMSOL matched the qualitative behavior of these experiments relatively well, especially for positions $\mathrm{B}$ and $\mathrm{C}$. The stationary model also matched previous nonconstrained photomechanical arch modeling efforts well $[42,43]$, along with experimental results of other materials systems currently under development.

For the constrained arch, the reflection symmetry of the standard arch is broken (Fig. 7b). In this case, the left-hand side of the constrained arch was initially buckled up and the light was focused in three different locations between the left-hand clamp and the pin. As a function of end displacement ratio, $T_{c r}$ has negative slope for the light focused at positions A and B. However, this slope becomes positive for the light position $\mathrm{C}$ (decreasing $T_{c r}$ with increasing end displacement). The reduced $T_{c r}$ at position C likely occurs in part because of its proximity to an inflection point where the flattening curvature facilitates the transition to snap-through.

In the experimental system (Fig. 7b) we see that end displacement and light position can be used to tune the latency times (or critical strain). The order of latency times follow the trend $\left(T_{c r}(\mathrm{C})<T_{c r}(\mathrm{~A})<\right.$ $\left.T_{c r}(\mathrm{~B})\right)$ for larger end displacements. For end displacement, $L / L_{o}=0.99$, the latency times follow $\left(T_{c r}(\mathrm{~A})\right.$ $<T_{c r}(\mathrm{~B})<T_{c r}(\mathrm{C})$ ), which is the same trend seen in simulations for all end displacements (Fig. S2). Another parameter that is easily adjusted is the pin height. Specific trends in $T_{c r}$ as a function of end displacement, when the pin was raised vertically by 200 um or lowered by $200 u m$, were ambiguous. However, when the pin was raised, all critical times were relatively short $(<110 \mathrm{~s})$, while lowering the pin resulted in critical times over 100s with many around 400s and a maximum of 1000s. In contrast to the experiments, simulations indicate that lowering the pin should have relatively little effect on the critical time (strain) until the pin is lowered more than 2 pin diameters (approximately 1400um in this case).

In general, many of the trends observed in experiments deviated from those observed in simulations. For example, the simulations predict that light focused near the clamped end can cause the material to lose contact with the pin and transition to an upward buckled, first mode shape. In the experiments, light focused closer to the clamp than position $\mathrm{C}$ usually results in no snap-through transition, and the strip maintains contact with the pin throughout irradiation. The absence of the upward buckled state 
in experiments is likely due to an attractive force between the pin and the azoPI strip (e.g. electrostatic attraction) as mentioned earlier. Indeed, even when the top of the pin is positioned 200um above the level of the clamps, the arch does not evolve into the standard first mode shape for a clamped-clamped beam, buckled upward. The fact that the system is stable in this configuration supports the claim that there is some small attractive force between the pin and the azoPI strip. Further, in the simulations it is observed that sliding of the arch along the pin reduces some of the induced strain and prevents the build up of sharp curvature regions like those observed in experiment. The ability or inability for the strip to slide along the pin or lose contact seems be a significant factor in constrained arch behavior. Additional experiments should be performed in the future with responsive material systems at scales in which this attractive force can be neglected in order to more fully correlate simulation and experiment.

\section{Conclusion}

$\mathrm{Bi}$ - and multistable arches constructed from responsive, slender elastic members with rigid lateral constraints represent a notable class of compliant mechanisms. In particular, these arches have the potential to be driven by light or other external stimuli and present a multitude of options for fine tuning system behavior through variations in stimuli and constraint characteristics. These arches are also intriguing because the nature of the motion is diversified from that of a standard single clamped-clamped arch. For example, snap-through due to irradiation on one side of an arch with a single constraint automatically leads to snap-through on the un-irradiated side as well. Because the arches in this work can be controlled solely with stimuli from above they may be useful as oscillating actuators or energy harvesters driven by thermal or chemical stimuli gradients. Further, their predicted response combines multistability with motions that feature discrete vertical, horizontal, and rotational characteristics. While the design motif in the present work features interesting qualitative mechanical behavior, the material system employed requires a lengthy relaxation process to reset the material with visible light or heat. Future work should focus on the development of materials that feature significant mechanical response and that relax quickly when the stimulus is removed [50,51]. Further, to increase actuation efficiency, parameters such as the focus point and width of the irradiation should be tailored to minimize the critical photostrain required for device operation. It should also be noted that the addition of an external constraint does add complexity to any future design concept. However, modular units could be devised such that constraints are incorporated into the support frame containing the clamps. Finally, conditions at the pin such as normal and frictional forces seem to significantly affect the azoPI arch behavior. Future work should explore these frictional effects, as well as, material systems and scales that minimize or harness them. The bi- and multistable arches presented here are ripe for further innovation and have the potential to impact energy harvesting, sensing, wireless actuation, and soft robotics applications.

\section{Acknowledgements}

The authors gratefully acknowledge support from the Materials and Manufacturing Directorate at the Air Force Research Laboratory and the Air Force Office of Scientific Research. JG and MRS also acknowledge support from the Office of Naval Research (N00014-18-1-2856) and the National Science Foundation (1635966). 


\section{References}

[1] Hu N and Burgeuño R 2015 Buckling-induced smart applications: recent advances and trends Smart Mater. Struct. 24063001

[2] Boisseau S, Despesse G, Monfray S, Puscasu O and Skotnicki T 2013 Semi-flexible bimetal-based thermal energy harvesters Smart Mater. Struct. 22025021

[3] Harne R L and Wang K W 2013 A review of the recent research on vibration energy harvesting via bistable systems Smart Mater. Struct. 22023001

[4] Hafez M, Lichter M D and Dubowsky S 2003 Optimized binary modular reconfigurable robotic devices IEEE ASME Trans. Mechatron. 8 18-25

[5] Howell L L, Magleby S P and Olsen B M 2013 Handbook of Compliant Mechanisms (John Wiley \& Sons)

[6] Forterre Y, Skotheim J M, Dumais J and Mahadevan L 2005 How the Venus flytrap snaps Nature 433 421-5

[7] Bennet-Clark H C and Daws A G 1999 Transduction of mechanical energy into sound energy in the cicada cyclochila australasiae J. Exp. Biol. 202 1803-17

[8] Smith M L, Yanega G M and Ruina A 2011 Elastic instability model of rapid beak closure in hummingbirds J. Theor. Biol. 282 41-51

[9] Oh Y S and Kota S 2009 Synthesis of Multistable Equilibrium Compliant Mechanisms Using Combinations of Bistable Mechanisms J. Mech. Des. 131021002

[10] Yu Y, Nakano M and Ikeda T 2003 Directed bending of a polymer film by light Nature 425145

[11] Jin Y, Paris S I M and Rack J J 2011 Bending Materials with Light: Photoreversible Macroscopic Deformations in a Disordered Polymer Adv. Mater. 23 4312-7

[12] Lee K M, Tabiryan N V, Bunning T J and White T J 2012 Photomechanical mechanism and structure-property considerations in the generation of photomechanical work in glassy, azobenzene liquid crystal polymer networks J Mater Chem 22 691-8

[13] Cviklinski J, Tajbakhsh A R and Terentjev E M 2002 UV isomerisation in nematic elastomers as a route to photo-mechanical transducer Eur Phys J E 9 427-34

[14] Kim T, Zhu L, Al-Kaysi R O and Bardeen C J 2014 Organic Photomechanical Materials ChemPhysChem 15 400-14

[15] Yamada M, Kondo M, Mamiya J, Yu Y, Kinoshita M, Barrett C J and Ikeda T 2008 Photomobile Polymer Materials: Towards Light-Driven Plastic Motors Angew. Chem. Int. Ed. 47 4986-8 
[16] Lee K M and White T J 2011 Photomechanical Response of Composite Structures Built from Azobenzene Liquid Crystal Polymer Networks Polymers 3 1447-57

[17] Skandani A A, Chatterjee S, Smith M L, Baranski J, Wang D H, Tan L-S, White T J and Shankar M R 2016 Discrete-state photomechanical actuators Extreme Mech. Lett. 9 45-54

[18] van Oosten C L, Bastiaansen C W M and Broer D J 2009 Printed artificial cilia from liquid-crystal network actuators modularly driven by light Nat. Mater. 8 677-682

[19] White T J, Tabiryan N V, Serak S V, Hrozhyk U A, Tondiglia V P, Koerner H, Vaia R A and Bunning T J 2008 A high frequency photodriven polymer oscillator Soft Matter 4 $1796-8$

[20] Gonçalves P B, Pamplona D, Teixeira P B C, Jerusalmi R L C, Cestari I A and Leirner A A 2003 Dynamic non-linear behavior and stability of a ventricular assist device Int. J. Solids Struct. 40 5017-35

[21] Tsuda T, Mochiyama H and Fujimoto H 2012 Quick stair-climbing using snap-through buckling of closed elastica 2012 International Symposium on Micro-NanoMechatronics and Human Science (MHS) 2012 International Symposium on Micro-NanoMechatronics and Human Science (MHS) (Nagoya, Aichi, Japan: IEEE) pp 368-73

[22] Gomez M, Moulton D E and Vella D 2017 Passive Control of Viscous Flow via Elastic Snap-Through Phys. Rev. Lett. 119

[23] Plaut R H and Virgin L N 2009 Vibration and Snap-Through of Bent Elastica Strips Subjected to End Rotations J. Appl. Mech. 76041011

[24] Gomez M, Moulton D E and Vella D 2017 Critical slowing down in purely elastic 'snapthrough' instabilities Nat. Phys. 13 142-5

[25] Sano T G and Wada H 2018 Snap-buckling in asymmetrically constrained elastic strips Phys. Rev. E 97013002

[26] Arena G, Groh R M J, Theunissen R, Weaver P M and Pirrera A 2018 Design and testing of a passively adaptive inlet Smart Mater. Struct. 27085019

[27] Zhu Y and Zu J W 2013 Enhanced buckled-beam piezoelectric energy harvesting using midpoint magnetic force Appl. Phys. Lett. 103041905

[28] Liu S, Hu N and Burgueño R 2015 Postbuckling Behavior of Axially-Compressed Strips With Discrete Rigid Constraints: A Numerical Study Development and Characterization of Multifunctional Materials; Mechanics and Behavior of Active Materials; Modeling, Simulation and Control of Adaptive Systems ASME Smart Materials, Adaptive Structures and Intelligent Systems vol 1 (Colorado Springs, CO USA) p V001T03A024

[29] Sano T G, Yamaguchi T and Wada H 2017 Slip Morphology of Elastic Strips on Frictional Rigid Substrates Phys. Rev. Lett. 118178001 
[30] Miller J T, Su T, Dussan V. E B, Pabon J, Wicks N, Bertoldi K and Reis P M 2015 Buckling-induced lock-up of a slender rod injected into a horizontal cylinder Int. J. Solids Struct. 72 153-64

[31] Jawed M K, Da F, Joo J, Grinspun E and Reis P M 2014 Coiling of elastic rods on rigid substrates Proc. Natl. Acad. Sci. 111 14663-8

[32] Marthelot J, Brun P-T, Jiménez F L and Reis P M 2017 Reversible patterning of spherical shells through constrained buckling Phys. Rev. Mater. 1025601

[33] He X Q, Wang C M and Lam K Y 1997 Analytical bending solutions of elastica with one end held while the other end portion slides on a friction support Arch. Appl. Mech. Ing. Arch. 67 543-54

[34] Plaut R H, Dillard D A and Borum A D 2011 Collapse of Heavy Cantilevered Elastica With Frictional Internal Support J. Appl. Mech. 78041011

[35] Wie J J, Wang D H, Lee K M, Tan L-S and White T J 2014 Molecular engineering of azobenzene-functionalized polyimides to enhance both photomechanical work and motion Chem Mater 26 5223-30

[36] Zhao Y and Ikeda T 2009 Smart Light-Responsive Materials (Hoboken, New Jersey USA: Wiley)

[37] Sankar B V and Tzeng, Jerome T 2002 Thermal Stresses in Functionally Graded Beams AIAA J. 40 1228-32

[38] Sankar B V 2001 An elasticity solution for functionally graded beams Compos. Sci. Technol. 61 689-96

[39] Jin L, Yan Y and Huo Y 2010 A gradient model of light-induced bending in photochromic liquid crystal elastomer and its nonlinear behaviors Int. J. Non-Linear Mech. 45 370-81

[40] Jin L, Lin Y and Huo Y 2011 A large deflection light-induced bending model for liquid crystal elastomers under uniform or non-uniform illumination Int. J. Solids Struct. $483232-$ 42

[41] Zhang Y and Huo Y 2018 First order shear strain beam theory for spontaneous bending of liquid crystal polymer strips Int. J. Solids Struct. 136-137 168-85

[42] Shankar M R, Smith M L, Tondiglia V P, Lee K M, McConney M E, Wang D H, Tan L-S and White T J 2013 Contactless, photoinitiated snap-through in azobenzene-functionalized polymers Proc. Natl. Acad. Sci. 110 18792-7

[43] Smith M L, Shankar M R, Backman R, Tondiglia V P, Lee K M, McConney M E, Wang D $\mathrm{H}$, Tan L-S and White T J 2014 Designing light responsive bistable arches for rapid, remotely triggered actuation Behavior and Mechanics of Multifunctional Materials and 
Composites SPIE Smart Structures and Materials + Nondestructive Evaluation and Health Monitoring vol 9058 (San Diego, CA USA) p 90580F

[44] van Oosten C L, Harris K D, Bastiaansen C W M and Broer D J 2007 Glassy photomechanical liquid-crystal network actuators for microscale devices Eur. Phys. J. E 23 329-36

[45] Warner M and Mahadevan L 2004 Photoinduced Deformations of Beams, Plates, and Films Phys. Rev. Lett. 92134302

[46] Skandani A A, Chatterjee S, Wang D H, Tan L-S, White T J, Shankar M R and Smith M L 2017 Relaxation dynamics and strain persistency of azobenzene-functionalized polymers and actuators Macromol. Mater. Eng. 3021700256

[47] Hogan P M, Tajbakhsh A R and Terentjev E M 2002 uv manipulation of order and macroscopic shape in nematic elastomers Phys. Rev. E 65041720

[48] Dunn M L 2007 Photomechanics of mono- and polydomain liquid crystal elastomer films J. Appl. Phys. 102013506

[49] Schneider C A, Rasband W S and Eliceiri K W 2012 NIH Image to ImageJ: 25 years of image analysis Nat. Methods $\mathbf{9} 671-5$

[50] Gelebart A H, Jan Mulder D, Varga M, Konya A, Vantomme G, Meijer E W, Selinger R L B and Broer D J 2017 Making waves in a photoactive polymer film Nature 546 632-6

[51] Gelebart A H, Vantomme G, Meijer E W and Broer D J 2017 Mastering the Photothermal Effect in Liquid Crystal Networks: A General Approach for Self-Sustained Mechanical Oscillators Adv. Mater. 291606712 